\title{
JUSTICIA AMBIENTAL
}

Revista Peruana Especializada en la Protección Jurídica del Ambiente del Poder Judicial

Vol. 1, n. ${ }^{\circ}$ 1, enero-diciembre, 2021, 13-24

Publicación anual. Lima, Perú

ISSN: 2810-8353 (En línea)

DOI: 10.35292/justiciaambiental.v1i1.457

\section{Análisis de la implementación de las políticas ambientales frente al compromiso de los ciudadanos y las autoridades}

\section{Analysis of the environmental policies implementation concerning the commitment of citizens and authorities}

Felipe Rafael Valle Díaz

Universidad Nacional José María Arguedas

(Apurímac, Perú)

Contacto: fvalle@unajma.edu.pe

https://orcid.org/oooo-0oo3-0855-9688

Rony Bequerel Méndez Soto

Corte Superior de Justicia de Lima

(Lima, Perú)

Contacto: rmendezs@pj.gob.pe

https://orcid.org/oooo-0oo3-1522-8756

\section{RESUMEN}

El objetivo general de la presente investigación es argumentar las condiciones de la implementación de las políticas ambientales frente al compromiso de los ciudadanos y las autoridades. Para este fin, se utiliza un enfoque cuantitativo, como diseño de investigación se emplea el estudio de caso, y el tipo de investigación es descriptivo. El escenario es el Ministerio del Ambiente, cuyo objeto de análisis son las acciones 
aprobadas y ejecutadas a nivel subnacional y local. La población y la muestra están compuestas por los informes y la memoria anual de entidades relacionadas con la implementación de la política ambiental. Se trata de una muestra no probabilística, conocida por conveniencia. Se verifica que existen considerables avances respecto de la mitigación ambiental, en sus tipos segregación de residuos sólidos y de aguas grises, y recuperación del deterioro de fajas marginales, que contribuyen a mejorar el estado de bienestar social; sin embargo, el crecimiento de la expansión urbana, que incluye el crecimiento de hogares en estado de necesidad y tugurización, produce que las brechas reducidas en el mediano plazo vuelvan a crecer en el corto plazo, porque la respuesta respecto de la mitigación ambiental toma períodos superiores para lograr cambios y madurar la cultura de conservación y protección ambiental; y el factor hombre presenta lenta adecuación, porque sus necesidades inmediatas están por encima de la cultura y de la conciencia ambiental que se pretende consolidar y desarrollar.

Palabras clave: implementación; políticas ambientales; compromiso; ciudadano.

\section{ABSTRACT}

This research presents the conditions of the implementation of environmental policies concerning the commitment of citizens and authorities. For this purpose, a case study is used as a research design, from a descriptive kind of research and a quantitative approach. The setting is the Ministry of the Environment, whose object of analysis is the actions approved and executed at the subnational and local levels. The population and the sample are composed of the annual report of the environmental policy implementation in the public institution. It is a non-probabilistic sample, known by convenience. The work shows that considerable progress has been made in environmental mitigation, in terms of solid waste and gray water segregation, and recovery of the deterioration of marginal strips. These improvements contribute to improving social welfare. However, it is also evident that the growth of urban sprawl, which includes the growth of households in a state of need and shantytowns, causes the gaps reduced in the medium term to 
grow again in the short term. Likewise, some reflections are analyzed, for example, that concerning environmental mitigation it takes longer periods to achieve changes and mature the culture of conservation and environmental protection; and concerning the human factor, there is a slow adaptation, because their immediate needs are above the culture and environmental awareness that is intended to consolidate and develop.

Key words: implementation; environmental policies; engagement; citizen.

Recibido: 19/10/2021 Aceptado: 10/11/2021

\section{INTRODUCCIÓN}

En el gobierno del expresidente Alan García Pérez, en el período 2007-2011 se realizaron las primeras reformas consensuadas a nivel del Centro Nacional de Planeamiento Estratégico (Ceplan) y el Ministerio de Educación (Minedu); se inició la reforma educativa basada en alcanzar las competencias y la meritocracia en el nivel básico, inicial, primaria y secundaria; para el caso del medio ambiente, se fortalecieron las políticas de intervención para minimizar impactos por factores antrópicos, que son los incidentes que determinan el deterioro de diversos ecosistemas, producidos por la explotación irracional que se disfraza de un aprovechamiento gradual. En este gobierno se creó el Ministerio del Ambiente (Minam), el cual articuló las intervenciones específicas a casos en situaciones de conflictos latentes y avanzados, además realizó inspecciones periódicas como parte de su competencia, con otros ministerios, como el de Energía y Minas, de Vivienda, Construcción y Saneamiento, entre otros.

Para el período de gobierno 2012-2016, del expresidente Ollanta Humala Tasso, se consolidaron las coordinaciones, el diseño de políticas, los programas y la capacidad de asignación presupuestal, para integrar y sujetarse a las recomendaciones hechas por el grupo de Desarrollo Sostenible para el Milenio, el Programa de Naciones Unidas para el Desarrollo (PNUD) y la Conferencia de las Partes (COP) (2015), cuyos objetivos buscaron poner fin al hambre y a la pobreza extrema, y promover la igualdad de género, reducir la mortalidad 
infantil, ampliar la calidad de vida con mayor dotación de servicios básicos, entre otros; sin embargo, resaltó que el Gobierno peruano incidiera en potenciar e incrementar con mayor interés las políticas de protección y conservación ambiental, lo cual se materializó en proporcionar recursos económicos, humanos y financieros en sectores clave, cuya conflictividad era alta, según reportes y estudios de la Defensoría del Pueblo peruana. A finales de este gobierno, se consolidó a nivel nacional, a través de los gobiernos locales, la implementación de programas presupuestales, como el manejo de residuos sólidos, la recuperación y mejora de fajas marginales de ríos, la mejora de suelos andinos, entre otros.

Como antecedente de competencia, se tienen las funciones del Organismo de Evaluación y Fiscalización Ambiental OEFA (2012), que realizó supervisiones de seguimiento y vigilancia del desempeño a los administrados, entre estos los más frecuentes fueron el sector minería mediana y grande: 136, e hidrocarburos: 234. Producto de las acciones de fiscalización ambiental, generaron 314 resoluciones directorales, de las cuales 134 fueron sobre sanciones, cuyo monto de imposición en multas ascendió a 20 179.67 UIT.

A partir del informe preelectoral elaborado por la Presidencia del Consejo de Ministros del Gobierno de Ollanta Humala, según el reporte del OEFA y el Ministerio del Ambiente (Minam, 2016), los resultados en política ambiental se reflejaron en la Agenda Nacional de Acción Ambiental al 2021, la cual busca alinear las acciones de las diversas instituciones que forman el Sistema Nacional de Gestión Ambiental, dando prioridad a las políticas públicas en el marco del Plan Bicentenario Perú al 2021. Los principales ejes son los siguientes:

1. La mitigación del cambio climático.

2. La promoción y el desarrollo de los recursos naturales y la diversidad biológica para un crecimiento sostenible.

3. La vigilancia para evitar acciones contaminantes.

Para el primer eje, según el Ministerio de Economía y Finanzas (2016), se organizó la COP 15, a la que acudieron más de 14 ooo personas; y se logró que el Fondo Verde para el Clima supere en 200 millones de dólares la meta trazada de capitalización inicial, que era 10000 millones de dólares. A nivel subnacional, en coordinación con el Minam, se logró institucionalizar la agenda climática para promover la implementación de planes de adaptación y mitigación frente al cambio climático (OEFA, s. f.). 
Respecto del segundo eje, se concretó la creación del Servicio Nacional de Certificación Ambiental para las Inversiones Sostenibles (Senace), cuya función principal es la evaluación y la aprobación de los estudios de impacto ambiental detallados (EIA-d) de los subsectores minería y energía, transferidas al Ministerio de Energía y Minas. Se diseñaron instrumentos técnicos para la certificación ambiental; respecto a la vigilancia, se aprobaron los marcos legales para frenar la minería y la tala ilegales. Son casos el Decreto Legislativo n. ${ }^{\circ} 1102$, sobre la tipificación del delito de minería ilegal; el Decreto Legislativo n. ${ }^{\circ} 1104$, sobre la pérdida de dominio; el Decreto Legislativo n. ${ }^{\circ} 1103$, sobre el control de insumos (mercurio, cianuro y combustibles); el Decreto Legislativo n. ${ }^{\circ} 1106$, sobre el lavado de activos; el Decreto Legislativo n. ${ }^{\circ} 1099$ y el Decreto Legislativo . $^{\circ} 1100$, sobre la interdicción de la minería ilegal a nivel nacional, entre otros (OEFA, s. f.).

Sobre el tercer eje, la gestión de la implementación del programa presupuestal para la Gestión Integral de Residuos Sólidos tuvo como objetivo disminuir la cantidad y la peligrosidad en el ambiente de residuos sólidos antrópicos, con una inversión de 101 millones de dólares, financiados por la Agencia de Cooperación Internacional del Japón (JICA) y el Banco Interamericano de Desarrollo (BID), que abarcan 31 proyectos de inversión pública (OEFA, s. f.).

A nivel nacional, las ciudades ubicadas en el vasto territorio peruano presentan dificultad extrema en el adecuado manejo de los residuos sólidos. Según Cahuas (2009), el problema se centra en el incremento de las zonas de expansión urbana, producto de la migración a partir de la década de los noventa hasta la primera década del siglo XXI; otro agente que catapultó el crecimiento urbano fue la formalización de la propiedad inmueble, en una primera etapa desde 1996 por el Proyecto Especial de Titulación de Tierras y Catastro Rural (PETT-CR), según Valle y Marín (2020) liquidado el 2007, y en una segunda etapa desde el 2003 hasta la actualidad por la Comisión de Formalización de la Propiedad Informal Urbana (Cofopri); y también se debe a la proximidad de territorios comunales inscritos que, por su cercanía a la ciudad, facilitan la conversión del uso del suelo y la habilitación urbana. En el caso de la sierra peruana, una gran cantidad de territorios comunales presentan este caso específico.

Valle y Marín (2020), respecto del estudio del cambio de uso del suelo y la expansión urbana, evidencian que desde los territorios comunales campesinos, 
como en los distritos de Andahuaylas y Anta, de la regiones de Apurímac y de Cusco, respectivamente, la formalización del suelo agrícola cercano a la zona urbana adquirió mayor valor. Las autoridades internas en los territorios comunales campesinos consolidaron el proceso a través de la generación de documentos imperfectos, que luego fueron legitimados y validados en la práctica por la población, a esto se suma que la legislación vigente presenta vacíos que son aprovechados para la conversión del suelo comunal a urbano.

Cahuas (2009) señala que en la sierra, alrededor de las ciudades capitales de las provincias y en menor intensidad en las ciudades capitales distritales, la propiedad privada en parcelas agrícolas ocupa una considerable extensión, y sus linderos se ubican en zonas urbano-marginales. Según el Ministerio de Agricultura y Riego (2015), el Proyecto Especial de Titulación de Tierras y Catastro Rural, conocido por las siglas PETT, mediante la herramienta de prescripción adquisitiva de dominio, formalizó un radical cambio de uso de suelo en cinturones marginales en la primera década del siglo XXI, generando considerables extensiones de tierra habilitadas para expansión urbana, en donde se establecieron familias de migrantes, quienes, conforme con los requisitos del Decreto Legislativo n. ${ }^{\circ}$ 667, permitieron la inscripción de la posesión de predios rurales próximos al área urbana, cuyo proceso de saneamiento físico-legal consolidaría la inscripción del derecho de propiedad.

A partir de este fenómeno social, según el Minam (2016), el incremento de residuos sólidos, a nivel nacional, fue manejado de modo regular, y trajo como consecuencia molestias a una considerable cantidad de familias cercanas, también botaderos informales, por la descomposición de materia orgánica, focos infecciosos de propagación de enfermedades, y desechos no clasificados que impedían el reúso. Además, existía, en la mayoría de gobiernos locales, un único botadero municipal, sin ningún plan de manejo y transformación de residuos sólidos. Esta realidad se mantiene hasta hoy, porque si bien hay un programa, la expansión urbana presenta un ritmo mayor al cual se le adiciona el incremento del número de miembros del hogar.

Para las ciudades de la sierra, como por ejemplo el gobierno local de Andahuaylas, se implementó un proyecto de mejora en el acopio de residuos sólidos, cuyo objetivo fue proporcionar bolsas degradables de plástico para basura a las familias ubicadas en los barrios o anexos donde la atomización de vivienda y tugurización de familias era de alto riesgo, es decir, aquellas viviendas 
de familias ubicadas en quebradas de profundidad extrema, laderas empinadas, cuyo único acceso eran escaleras comunes (Gerencia de Desarrollo Económico, Social y Medio Ambiente, 2015).

Las familias entregaban a los técnicos recolectores programados y contratados por la Municipalidad Provincial de Andahuaylas las bolsas de basura en horarios determinados. Este proyecto tuvo una aplicación de cinco años, desde el 2008 hasta el 2012. Los resultados fueron considerables, y se consiguió un 60 \% de eficacia; sin embargo, hubo un incremento de costos para el acopio y el traslado del residuo sólido, el cual alcanzó un 40 \% de eficiencia (Gerencia de Desarrollo Económico, Social y Medio Ambiente, 2015).

Después de ser aprobado el proyecto de inversión en diversas municipalidades provinciales a nivel nacional, los gobiernos locales aprobaron en sesión de concejo la oficialización del incremento de presupuesto y actividades a la Gerencia de Servicios y Medio Ambiente, lo cual hizo posible que, a mediados de 2016, se implementara el proyecto en mención en varios municipios provinciales (Minam, 2016).

A la fecha se sigue con la programación de actividades del programa presupuestal de mejora del servicio de manejo de residuos sólidos, que consta de centros de acopio oficiales, la adecuación de estos, kits clasificadores para el acopio de residuos sólidos, adquisición y uso de nuevos camiones compactadores para el recojo de basura (Gerencia de Desarrollo Económico, Social y Medio Ambiente, 2015).

A causa de la expansión urbana, se presentan inconvenientes en los recorridos del camión recolector, debido a las malas condiciones de transitabilidad en el espacio de la vía y las limitaciones de acceso para este; además de dificultades como la asignación de personal a áreas o sectores con problemas de limpieza y recolección de residuos sólidos, el mantenimiento de las unidades de recojo y acopio de residuos sólidos, la indiferencia de muchos hogares, próximos al centro de la ciudad, en los cinturones urbano marginales y en las expansiones urbanas no habilitadas, que presentan una cultura de expulsión de residuos en cualquier lugar, lo cual contribuye a la consolidación de focos infecciosos, contaminación odorífera, calles sucias, y perjudica la salud de los pobladores (GSMA-MPA, 2018).

Según la Gerencia de Desarrollo Económico, Social y Medio Ambiente (2015), en estas zonas existe inconsciencia de gran parte de la población, a pesar de ello, ante la observación y las entrevistas, la respuesta común es que 
la basura no puede quedarse en el interior de las viviendas, sino que debe ser expulsada a la calle. Esta conducta individualista que demuestra la cultura de muchos hogares, es el primer problema. El segundo es respecto a la labor del personal de limpieza del ornato público, el cual presenta limitaciones, se rigen de las disposiciones directas del inmediato superior, tienen estrictos horarios laborales e incluso son destinados hacia otros servicios de forma temporal.

Dado el estudio, el objetivo general de la investigación es argumentar las condiciones en la implementación de las políticas ambientales frente al compromiso de ciudadanos y autoridades.

El estudio permitirá detallar los avances en la implementación de la política ambiental en cuanto a la mitigación de la segregación de residuos sólidos y de aguas grises, a partir de la intervención de las decisiones políticas para alcanzar los objetivos y los compromisos planteados por el Estado peruano frente a la agenda ambiental, con el compromiso de la ciudadanía y la autoridad local.

\section{METODOLOGÍA}

Como diseño de investigación se emplea el estudio de caso, que describe y explica los elementos que integran el sistema social o las organizaciones, de forma holística, y está ubicado en el enfoque cuantitativo (Hernández, Baptista y Fernández, 2014, p. 164). Según Monje (2011), el paradigma cuantitativo se nutre del desarrollo de análisis de un marco teórico acotado y de la comparación apoyada en datos, para explicar los fenómenos con el método deductivo, el cual nos lleva a construir reflexiones desde los significados específicos (las acciones) hacia lo general, que es el logro obtenido (pp. 11-12).

En cuanto al diseño de investigación, abarcamos un período específico para realizar una evaluación del proceso de implementación de la política ambiental a partir de la toma de decisiones, y un examen sistemático profundo de los diversos aspectos en la entidad social (Monje, 2011, p. 117). El tipo de investigación que se utiliza es la descriptiva, para estudiar la institución y la toma de decisiones respecto de la implementación de políticas, ya que el objeto es analizado bajo la premisa y las categorías del método de la narración e ilustración, que permite afianzar y sostener la implementación de la toma de decisiones, además de acompañar con figuras y croquis para profundizar la comprensión (Hernández, Baptista y Fernández, 2014, pp. 10-11). 
La población y la muestra están compuestas por los informes, la memoria anual de las direcciones que conforman el Ministerio del Ambiente, los reportes de agencias de cooperantes sobre el medio ambiente y los informes de los municipios locales. Es una muestra no probabilística, conocida por conveniencia (Rodríguez, Gil y García, 1996).

Las técnicas y los instrumentos de recolección de datos que se utilizan son los siguientes: la revisión de literatura documentaria, cuyo instrumento es la lista de resumen, porque desarrollamos síntesis aproximadas y referenciadas para consignarlas en el marco teórico referencial; y la comparación, cuyo instrumento es una tabla de doble entrada, que permitirá esbozar los aspectos primordiales de cada indicador a partir de la argumentación de los diagnósticos, para obtener diferencias probadas considerables (Monje, 2011, pp. 151-154).

\section{RESULTADOS}

Los resultados se obtuvieron a partir del cruce de información, el análisis y la depuración de informes a partir de la coordinación y ejecución a nivel subnacional dentro del territorio peruano, bajo jurisdicción del Estado.

A nivel de los gobiernos subnacionales, podemos establecer que las gerencias regionales del medio ambiente iniciaron la programación y la ejecución de los planes y las directrices sectoriales, e implementaron una importante cantidad de programas y proyectos. Asimismo, las oficinas de evaluación y fiscalización ambiental (OEFA), cuyos roles son ser reguladores, dictaminadores y sancionadores, tuvieron importantes logros, al sancionar a infractores reincidentes que recayeron con mayor frecuencia en el sector minero e industrial; este rol se les retiró hace tres años.

Los logros a la fecha son los siguientes:

1. En diez regiones calificadas con alto riesgo de incidencia del calentamiento global, ubicadas en la sierra peruana, han implementado recursos para ampliar la cobertura forestal en pisos altitudinales entre los 3000 a $3600 \mathrm{~m}$ s. n. m. Entre estas, sobresalen por sus avances y logros Arequipa, Ayacucho, Apurímac, Cusco y Huancavelica.

2. $\mathrm{Al}$ 2020, aproximadamente 600 municipios, a nivel del territorio nacional peruano, cuentan con un programa de ciudadanía ambiental, conocido como Educca, que consolida el Plan Nacional de Educación Ambiental 2017-2022. 
3. Los gobiernos subnacionales, ubicados en el Perú, cuentan con un sistema de información ambiental incorporado al Sistema Nacional de Información Ambiental (Sinia), que sirve como soporte para el programa Educca.

4. Los gobiernos subnacionales cuentan con agendas ambientales de investigación, cuyos componentes y objetivos están articulados a los sectores agricultura, turismo, cultura, minería, entre otros.

5. $\mathrm{Al}$ 2020, aproximadamente 460 municipios, de preferencia provinciales, a nivel del territorio nacional peruano, han implementado proyectos de segregación en la fuente de residuos sólidos, destinados a mejorar el relleno sanitario, la cadena de recolección de residuos sólidos, la selección y el reciclado de estos, y su tratamiento y transformación.

6. Cuatro gobiernos subnacionales han apoyado al gobierno nacional, a nivel provincial, específicamente a las empresas de saneamiento básico, para implementar e invertir progresivamente en plantas de tratamiento de aguas grises, de tal forma que esta decisión sea un ejemplo y se minimice el impacto ambiental en los ríos de la vertiente occidental, contenidos en el territorio peruano.

\section{CONCLUSIÓN}

Los esfuerzos y la habilitación de recursos económicos, humanos y financieros, a partir de los entes rectores de la política peruana, los órganos ejecutores subnacionales y locales encargados de la implementación de programas y proyectos ambientales, además del apoyo internacional, han hecho posible que se tenga un considerable avance respecto de la mitigación del impacto ambiental en sus tipos segregación de residuos sólidos, de aguas grises, recuperación del deterioro de fajas marginales, los cuales contribuyen a mejorar el estado de bienestar social; sin embargo, el problema del crecimiento de la expansión urbana, que incluye el crecimiento de hogares en estado de necesidad y tugurización, sostenida en vacíos legales y que son aprovechados para fortalecer el cambio de uso de suelo y la habilitación urbana, hace que las brechas reducidas en el mediano plazo vuelvan a crecer en el corto plazo, porque la respuesta respecto de la mitigación ambiental toma períodos superiores para lograr cambios y madurar la cultura de conservación y protección ambiental. El 
factor antrópico presenta lenta adecuación, pues sus necesidades inmediatas están por encima de la cultura y la conciencia ambiental que se pretende consolidar y desarrollar.

\section{REFERENCIAS}

Cahuas, P. J. (2009). Proyecto especial de titulación de tierras (PETT) en Ilave-Puno [Informe de experiencia profesional]. Universidad Nacional Agraria de la Selva.

Gerencia de Desarrollo Económico, Social y Medio Ambiente. División de Gestión del Medio Ambiente (2015). Programa de segregación en la fuente y recolección selectiva de residuos sólidos en el distrito de Andahuaylas 2015. http://siar.minam.gob.pe/apurimac/documentos/programa-segre gacion-fuente-recoleccion-selectiva-residuos-solidos-1

Hernández, R., Baptista, P. y Fernández, L. (2014). Metodología de la investigación. McGraw Hill.

Ministerio de Agricultura y Riego (2015). Informe de resultados del proceso de titulación de los predios rurales.

Ministerio de Economía y Finanzas (2016, 25 de septiembre). Informe PreElectoral 2011-2016. https://www.gob.pe/institucion/mef/informespublicaciones/1477414-informe-pre-electoral-2011-2016

Ministerio del Ambiente (Minam) (2016). Memoria Anual 2016. https://www. minam.gob.pe/wp-content/uploads/2017/o6/Memoria-Anual-2016MINAM.pdf

Monje, C. A. (2011). Metodología de la investigación cuantitativa y cualitativa. Guía didáctica. Universidad Surcolombiana.

Organismo de Evaluación y Fiscalización Ambiental (OEFA) (s. f.). El Organismo de Evaluación y Fiscalización Ambiental (OEFA) y el macroproceso de fiscalización ambiental: logros enero-julio de 2012. https://www.oefa. gob.pe/portada/estadisticas-y-resultados/ 
Rodríguez, G., Gil, J. y García, E. (1996). Metodología de la investigación cualitativa. Aljibe.

Valle, F. R. y Marín, C. (2020). Uso de la tierra y expansión urbana en las comunidades campesinas de los distritos de Andahuaylas y Anta, regiones Apurímac y Cusco, Perú. Ciencia Latina. Revista Multidisciplinar, 4(2), 431-450. https://doi.org/10.37811/cl_rcm.v4i2.90 